\title{
Treatment of Complex Regional Pain Syndrome (CRPS) Using Low Dose Naltrexone (LDN)
}

\author{
Pradeep Chopra • Mark S. Cooper
}

Received: 7 November 2012 / Accepted: 4 March 2013 /Published online: 2 April 2013

(C) The Author(s) 2013. This article is published with open access at Springerlink.com

\begin{abstract}
Complex Regional Pain Syndrome (CRPS) is a neuropathic pain syndrome, which involves glial activation and central sensitization in the central nervous system. Here, we describe positive outcomes of two CRPS patients, after they were treated with low-dose naltrexone (a glial attenuator), in combination with other CRPS therapies. Prominent CRPS symptoms remitted in these two patients, including dystonic spasms and fixed dystonia (respectively), following treatment with low-dose naltrexone (LDN). LDN, which is known to antagonize the Toll-like Receptor 4 pathway and attenuate activated microglia, was utilized in these patients after conventional CRPS pharmacotherapy failed to suppress their recalcitrant CRPS symptoms.
\end{abstract}

Keywords Chronic pain - Complex regional pain syndrome $\cdot$ CRPS $\cdot$ Reflex sympathetic dystrophy $\cdot$ RSD . Neuropathic pain $\cdot$ Naltrexone $\cdot$ Fixed dystonia $\cdot$ Allodynia . Vasomotor · Ulceration · Dystonic spasms · Conversion disorder $\cdot$ Functional movement disorder $\cdot$ LDN

\section{Introduction}

Complex Regional Pain Syndrome (CRPS), formerly known as Reflex Sympathetic Dystrophy (RSD) is a neuroinflammatory condition that is characterized by a combination of sensory, autonomic, vasomotor, and motors

P. Chopra $(\bowtie)$

Department of Medicine, Alpert Medical School of Brown University, 102 Smithfield Ave,

Pawtucket, RI 02860, USA

e-mail: painri@yahoo.com

M. S. Cooper

Department of Biology, Graduate Program in Neurobiology

and Behavior, University of Washington, Seattle,

WA 98195-1800, USA

e-mail: mscooper@u.washington.edu dysfunctions. One of the characteristic symptoms of this condition is that the pain is out of proportion to the initial injury. Diagnoses of CRPS are often delayed because it is under recognized (Binkley 2012). If effective treatments are given early enough in progression of the disease, there is reduced chance for the spread of regional pain, autonomic dysfunction, motor changes, and negative sensory symptoms, such as hypoalgesia (Marinus et al. 2011). As CRPS progresses, it becomes refractory to sympathetic nerve blocks, conventional analgesics, anticonvulsants and antidepressants.

During neuroimmune activation, TLR4 (Toll-Like Receptor 4) is upregulated in microglia, resident immune cells of the central nervous system (Watkins et al. 2009). After transection of the L5 spinal nerve in the rat, TLR4 expression is increased in spinal microglia. This correlates with the rodent developing neuropathic pain (Tanga et al. 2005). From a postmortem analysis of a CRPS patient, activated microglia and astroglia in the central nervous system (CNS) have been implicated in the generation of CRPS symptoms (Del Valle et al. 2009).

Activation of TLR4 in both microglia and CNS neurons augments the production of pro-inflammatory cytokines via the NF-kB pathway (Milligan and Watkins 2009; LeowDyke et al. 2012). NF-kB is a multi-functional transcription factor that is activated by c-Jun-N-terminal kinase (JNK), extracellular signal-related kinase (ERK), or p38 (Milligan and Watkins 2009). In activated glia and neurons, NF-KB activity promotes the production of pro-inflammatory cytokines and neurotoxic superoxides (Milligan and Watkins 2009; Leow-Dyke et al. 2012; Fellner et al. 2013), which act as mediators for neuropathic pain, as well as other neurological dysfunctions (Liu et al. 2000; Barbosa et al. 2012; Besedovsky and del Rey 2011). Pro-inflammatory cytokines, as well as the neurotrophin BNDF (brain-derived neurotrophic factor), induce enhanced excitatory tone and diminished inhibitory tone in nociceptive neural networks, 
leading to hyperalgesia or allodynia (von Hehn et al. 2012). Sustained TLR4 stimulation in microglial populations can also lead to neuronal injury and death (Fellner et al. 2013).

In rodents, the TLR4 antagonist, naltrexone, is able to suppress allodynia arising from bone cancer (Mao-Ying et al. 2012). In rodents, naltrexone is able to cross the bloodbrain barrier, suppress glial cell activation, and reverse neuropathic pain arising from chronic constriction nerve injury (Hutchinson et al. 2008).

A recent study reports that low-dose oral naltrexone reduces pain in fibromyalgia patients (Younger and Mackey 2009). Low-dose naltrexone (LDN) refers to doses approximately 50-fold lower than doses of naltrexone typically given to patients addicted to opioids (Rea et al. 2004; Younger and Mackey 2009).

Opiate antagonists differ from opioids through a replacement of the characteristic N-methyl group with a $\mathrm{N}$-cyclopropyl, $\mathrm{N}$-allyl group. At low concentrations, naltrexone is able to antagonize TLR4 on activated glial cells, without inhibiting other opioid receptors in the CNS (Hutchinson et al. 2008). This allows endogenous anti-nociceptive pathways involving $\mu$-receptors to continue operating.

A recent paper has reported positive benefit of ibudilast, an oral glial attenuator, for the treatment of neuropathic pain in several CRPS patients (Rolan et al. 2009). Below, we describe positive outcomes of two CRPS patients treated with low-dose naltrexone, in combination with other CRPS therapies. Low-dose naltrexone was utilized in these patients after more conventional CRPS pharmacotherapy failed to suppress their recalcitrant CRPS symptoms. Each patient met IASP criteria for the diagnosis of CRPS.

\section{Case 1. Clinical course and treatment}

A 48 year-old male veteran sustained an injury to his right leg in 2006. While undergoing aqua-therapy, he injured his right great toe, which subsequently became infected. Following drainage of an abscess and removal of the great toe's nail, the patient developed the following CRPS symptoms in his right lower extremity: swelling, allodynia (pain to normal touch), color change, temperature change, and some weakness. By 2007, the patient developed moderate CRPS symptoms in his upper extremities. In 2008, he developed blisters and skin ulceration in his right lower extremity (Fig. 1). At this time, the patient was being treated with opioids, pregabalin, and duloxetine.

By 2009, the patient's pain had become severe enough that he could not ambulate without assistance. He developed muscle spasms in the right upper extremity. In 2010, he underwent a cardiac bypass surgery for coronary artery disease. His CRPS symptoms became widespread after this surgery, spreading to his upper chest, upper arms, and forearms. In 2011, the patient developed significant dystonic spasms to both upper extremities, resulting in hyperextension of his fingers. From 2008 to 2012, the patient underwent multiple treatments with anticonvulsants, antidepressants, physical therapy, psychotherapy, topical and systemic analgesics, including but not limited to opioids. The patient also currently has diabetes mellitus type II, hypertension, hyperlipidemia, as well as coronary artery disease.

In August of 2011, the patient began low-dose intravenous ketamine infusions. He reported a good initial response, but the relief was not sustained. He continued to have ketamine booster infusions at intervals of 4 to 6 weeks. Although the patient engaged in aggressive physical therapy during this time, his maximum interval of relief from pain following a given ketamine infusion decreased to an interval of 3 weeks. In January 2012, his use of the opioid oxycodone was changed to tapentadol. This narcotic was removed for 1 week prior to starting low-dose naltrexone, which was started and maintained at $4.5 \mathrm{mg}$ per day (1 dose at night). Additional medications included: metformin, tramadol, valsartan, cloazepate, simvastatin, fish oil, and vitamin C.

Immediately before LDN treatment, the patient had patchy areas of allodynia to the medial and dorsal aspect of his right foot, extensive areas of dysesthesia in his right lower extremity below the knee and heel of his foot, as well as bilaterally dysesthesia in the upper extremities. There were significant color and temperature changes in the right foot compared to the left foot, as well as pitting edema in the right foot. A triple-phase bone scan revealed significant reuptake in the right foot, characteristic of CRPS.

By March of 2012, the patient's requirements for the lower dose intravenous ketamine infusions were not as frequent (6 week intervals, pain spikes not as high). The patient recovered from CRPS flares more quickly, felt more energetic, and tolerated pain better. He became physically more active, and his sleep improved significantly. Within 2 months after starting LDN, the patient's dystonic spasms discontinued, although he still had moderate pain in both upper extremities. The patient was able to walk without a cane (Fig. 2b), which he had used continuously since 2006. His pain was an average of 8 to 10 on the Numeric Rating Scale (NRS) before starting LDN. It dropped down to an average of 5 to 6 on the NRS after starting LDN. After LDN therapy, the patient's pain symptoms have reduced in severity, but not in their distribution. His current mood state is good. No side effects of LDN were noted.

\section{Case 2. Clinical course and treatment}

A female patient, currently 12 years old, has a genetic disorder, Ehlers-Danlos Syndrome (EDS) hypermobility type 1, dysautonomia, non-epileptic seizures, chronic gastritis, mitochondrial dysfunction, asthma, vision loss, thyroid tumor 
Fig. 1 Advanced CRPS symptoms in Case 1. 4.5 years after onset of the disorder. a Allodynia and pronounced vasomotor dysfunction are present in the lower right extremity (photo taken 9/18/ 2008). b One week later, the leg has developed numerous ulcerations
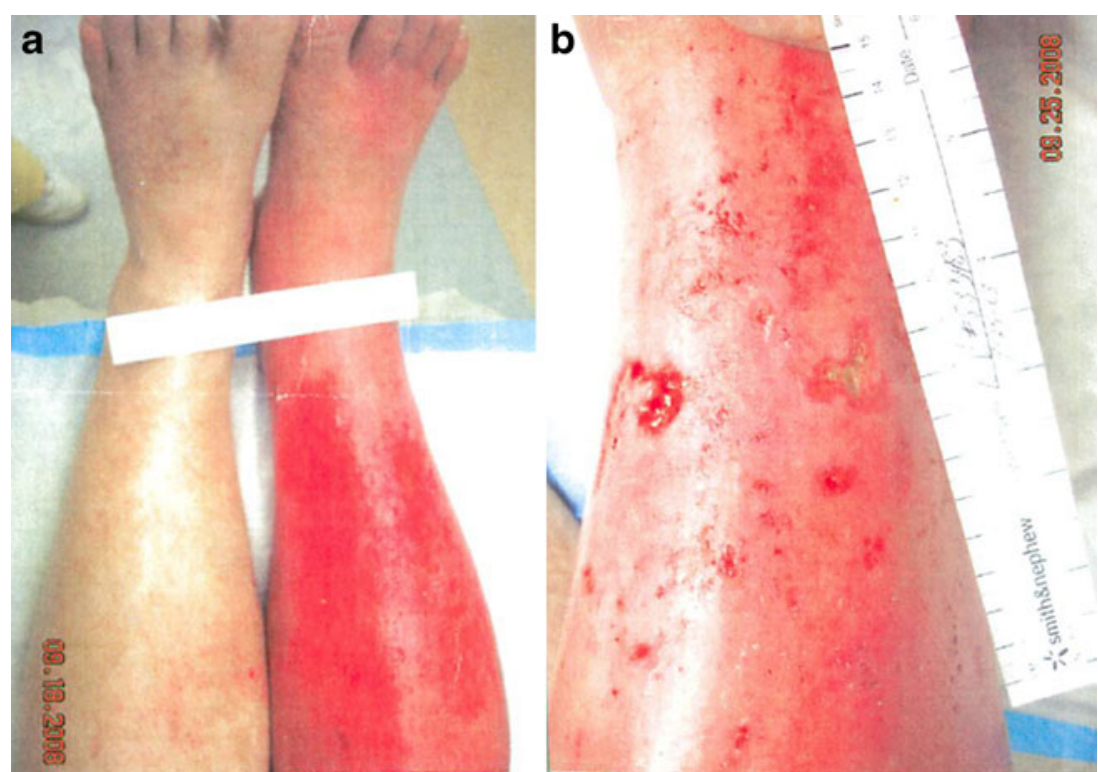

and anti-cardiolipin antibodies. As a result of her EDS, the patient has had repeated dislocations of her right shoulder, as well as her right ankle. The patient first developed CRPS in her lower right extremity in 2008. In 2009, the patient developed dystonic muscle spasms in the upper extremities, which were interpreted by her physicians as evidence of a conversion disorder.

The patient was first seen by one of us (PC) in February of 2011. Her symptoms of CRPS included color change, increased temperature in the right foot $\left(90^{\circ} \mathrm{C}\right)$ versus $\left(82^{\circ} \mathrm{C}\right)$ in the left foot, allodynia over the dorsum of right foot, and patchy areas of dysesthesia over her right leg. She also had areas of allodynia and dysesthesia in the left lower extremity and both upper extremities, as well as distorted nails on the right foot. When first seen, the patient's medications were levetiracetam, midodrine, baclofen, trazodone, diphenhydramine, lansoprazole, budesonide, levalbuterol, L-Carnitine, coenzyme Q10, vitamin D, vitamin C, magnesium, and vitamin B complex. She rated her pain on a NRS (Numerical Rating Scale) as 8/10 at its lowest and 10/10 at its worst. In early 2011, the patient experienced a subluxation of her right ankle. The lower right leg and ankle subsequently developed fixed dystonia, with plantar flexion and varus (Fig. 3a).

In June 2011, the patient was started on low dose naltrexone (LDN) of $3 \mathrm{mg}$ once a day and ketamine troches (sublingual) $10 \mathrm{mg}$ on as needed basis. The LDN was increased to $4.5 \mathrm{mg}$ per day, 4 weeks after starting it. LDN was started as a lower dose to gauge tolerability. She tolerated the LDN very well with
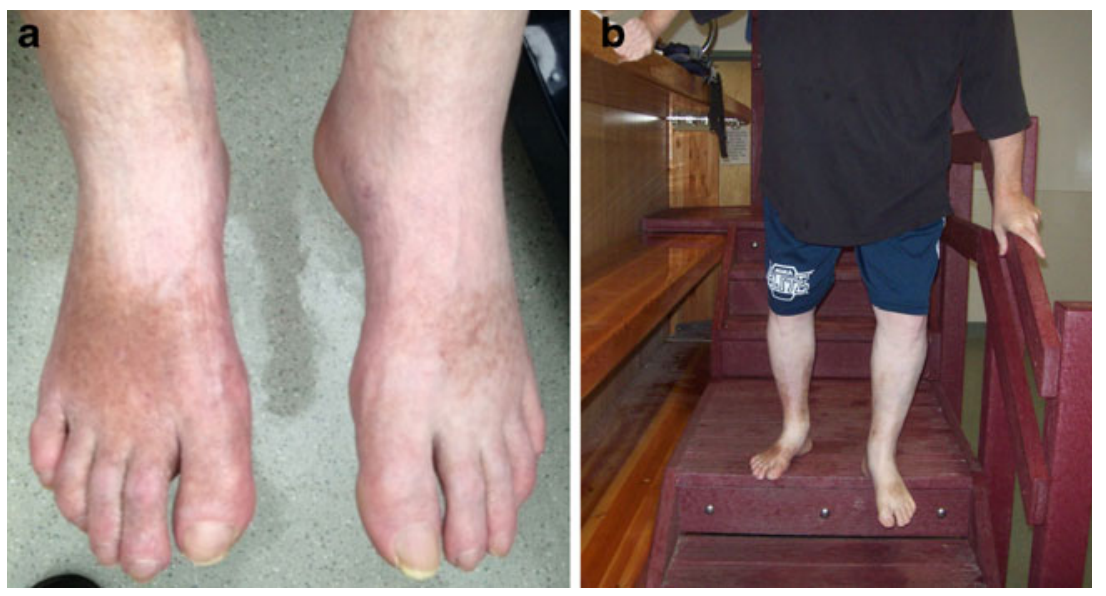

Fig. 2 Case 1. Certain symptoms are attenuated following treatment with low-dose naltrexone (LDN) in a long-standing case of CRPS (6 years after onset). a Allodynia is greatly reduced in both legs after LDN. However, bilateral trophic changes remain in the lower extremities. Slight swelling is present in the distal portion of the right foot.
Within 2 months of treatment with LDN, the patient was able to bear full body weight, and walk without assistance. Before LDN, the patient utilized a cane for 6 years. b One year after LDN treatment, the patient still has persistent long-term trophic changes in the skin of both lower extremities (taken 1/16/2013) 
Fig. 3 (Case 2) Fixed dystonia, allodynia, and vasomotor abnormalities in the right lower extremity of a CRPS patient (panel a) remitted following treatment with a low-dose naltrexone. b No symptoms and signs of CRPS after LDN treatment. Two months after surgical reinforcement of the right ankle for Ehlers-Danlos Syndrome (EDS)
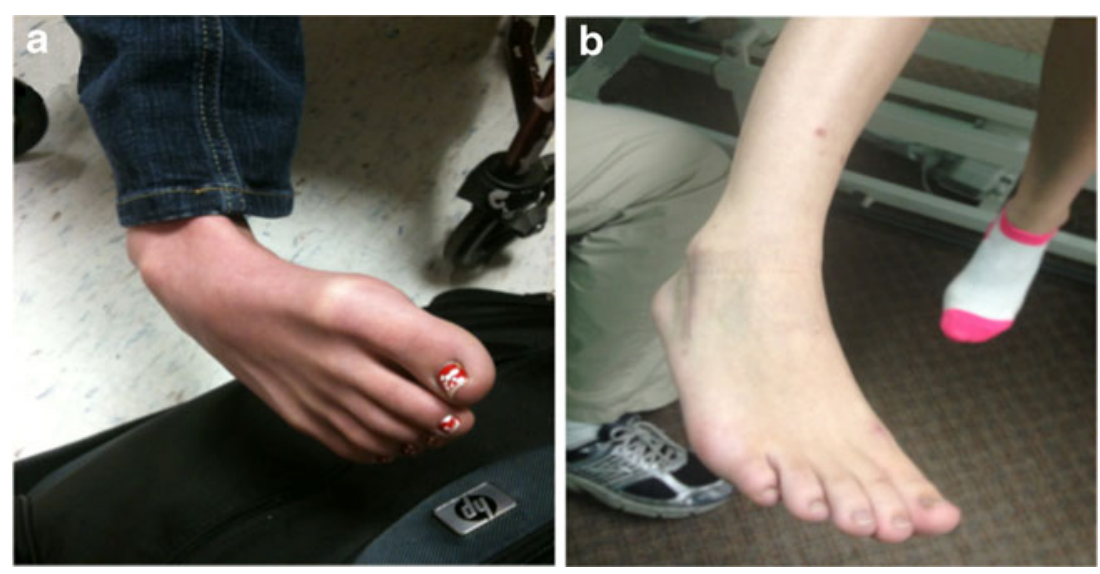

no reports of adverse effects. Her pain scores dropped from NRS (7-10)/10, to (3-5)/10. She also reported a decrease in allodynia, as well as a decrease in sensitivity to touch and temperature change. There was no effect on the dystonia. Two months after starting LDN, she underwent 400 units of botulinum toxin A injections to the right gastrocnemius group of muscles with minimal relief of her dystonia. The right lower extremity was placed in an above knee cast for 6 weeks. Several weeks after the cast was removed, the patient subluxated her right ankle again. She underwent a 400 units injection of botulinum toxin, and a cast was applied again. The patient used ketamine troches $(10 \mathrm{mg})$ for 3 weeks after the LDN was started, as needed for pain. She was taking ketamine troches on as needed basis for acute flare-ups of her CRPS pain. This was limited to no more than $30 \mathrm{mg}$ of ketamine troches per day. The patient progressively reduced her use of ketamine troches 3 weeks after starting LDN, and by 8 weeks was taking them rarely.

In December of 2011, she underwent percutaneous pinning of the right ankle to stabilize the joint. The pins were removed 6 weeks later and the ankle joint subluxated again. In February of 2012, the patient underwent reinforcement to the right ankle with cadaver ligaments. She continued to be on low dose naltrexone during this time, except for 4 perioperative days, during which the LDN was discontinued. As per institutional protocol, LDN was stopped for $24 \mathrm{~h}$ before surgery. After surgery, she was given oral opioids (hydrocodone with acetaminophen) for 1 week. The oral opioids were then discontinued, and LDN treatment was resumed $24 \mathrm{~h}$, after her last dose of opioid. The patient noticed a decrease in her post-operative pain, ranging from (4-6)/10, 3 weeks after resuming LDN. Skin discoloration and allodynia could not be assessed after the surgery because her leg was in a cast.

At the time of cast removal, the patient's lower leg and ankle had a normal range of motion, indicating a remission of the leg's fixed dystonia (Fig. 3b). The leg had a slight red color at the time of cast removal, but no allodynia. Ten months after surgery, the patient's gait was normal.
Remarkably, the patient did not experience any spread of her CRPS, despite undergoing multiple invasive procedures, including surgery. One of the known triggers for a CRPS flare-up, or spread of CRPS symptoms, is trauma (Schwartzman et al. 2009; van Rijn et al. 2011). The patient still has a chronic shoulder dislocation, associated with her EDS. However, her CRPS symptoms have resolved completely. The patient has been maintained on LDN for 18 months. No side effects of LDN have been noted.

\section{Discussion}

Causalgia, which is often a salient feature of CRPS, has long been viewed as having a neuroinflammatory etiology (Mitchell 1872; Sudeck 1901). Although centralized neuropathic pain has been connected to activated glia in rodents (Milligan and Watkins 2009), it is an open question whether the symptoms in CRPS in humans are linked to activated glia in the CNS. The positive responses of the two patients discussed above to LDN are among the first reported benefits of glial attenuators for CRPS symptoms. A prior study described moderate pain benefit to several CRPS patients enrolled in a clinical trial of ibudilast, another glial attenuator that is unrelated to LDN in its molecular action (Rolan et al. 2009).

In the patient of Case Study 1, dystonic spasms developed in the upper extremities 1 year after the onset of CRPS in the right lower extremity. The spread of CRPS symptoms, including movement disorders, may involve a spread of neuroinflammation within the neuraxis (Del Valle et al. 2009; Cooper 2011; Cooper and Clark 2012). Altered cytokine involvement has also been postulated to contribute to the spread of symptoms in CRPS patients (Linnman et al. 2012). Distortion of somatotopic maps (Maihöfner et al. 2003, 2004), deficient drive from the parietal cortex to the supplemental motor and primary motor corticies (Maihöfner and Peltz 2011), bilateral disinhibition of intracortical inhibition (Schwenkreis et al. 2003), disinhibited spinal 
reflexes, and paresis of affected extremities, are known functional impairments of CRPS patients (Birklein et al. 2000; van Hilten et al. 2001, 2011; Schouten et al. 2003; van Hilten 2010; Kolb et al. 2012). CNS neuroinflammation has also been postulated to generate functional movement disorders in inflammatory neurological disorders other than CRPS (Church et al. 2004; Sansing et al. 2007; van de Warrenburg et al. 2007). By attenuating activated CNS glia systemically, LDN may be especially useful for the treatment of CRPS symptoms, some of which might be generated by spreading neuroinflammation within the neuraxis (e.g. fixed dystonias, Cooper and Clark 2012).

In Case 1, repeated ketamine infusions were not adequate to drive the patient's CRPS symptoms into remission. Therefore, LDN was added to his polypharmacy. Ketamine blocks NMDA glutamate receptors, whereas LDN acts on Toll-Like Receptors in activated glia. These two medications would not be expected to interact at similar molecular sites, but would be expected to complement each other actions in suppressing hyperexcitable nociceptive neurons. Ketamine would be expected to act rapidly and directly on NMDA receptors in glutamatergic synapses, whereas LDN would be expected to act more slowly and indirectly by suppressing the neuroinflammatory activities of activated glia, which alter the excitability of nociceptive neuronal networks through the release of sensitizing agents, such as the neurotrophin BDNF (Coull et al. 2005).

In neurons, extracellular signal-related kinase (ERK) is activated through the TLR4 pathway. ERK can further sensitize excited AMPA and NMDA receptors, leading to neuropathic pain (Milligan and Watkins 2009). By attenuating TLR4 signaling, LDN could have an indirect inhibitory action on the activity of AMPA and NMDA receptors in sensitized glutamate-receptive neurons.

NMDA receptors (NMDARs) are also present in activated microglia. NMDARs stimulation can activate microglia in vitro, as well as contribute to their neuroinflammatory response (Kaindl et al. 2012). In human patients with centralized neuroinflammation, such as CRPS (del Valle et al. 2009), a therapeutic combination of ketamine and LDN (through their respective ionotrophic and metabotrophic actions) might synergistically suppress the pro-inflammation paracrine signaling activities of activated microglia.

Glial attenuators are likely to provide benefits in controlling CNS neuroinflammation that arises from periphery nerve injury, CNS injury, or autoimmune attack on CNS targets. However, it is possible that inhibiting CNS neuroinflammation with glial attenuators might be deleterious to immuno-compromised individuals, or individuals with an ongoing viral or bacterial infection. However, naltrexone has been used in HVC and HIV patients to treat opioid addiction, without causing major complications (Mitchell et al. 2012). In general, before utilizing LDN in a given individual, practitioners should be aware that atypical cases of CRPS and movement disorders have been linked to infectious agents, such as Borrelia burgdorferi (Lyme disease) (Sibanc and Lesnicar 2002) and parvovirus B19 (Fong and de Sousa 2006; Grillo and da Silva 2009). The safety of using LDN to attenuate neuroinflammation, pain, and movement disorders, in these particular infectious diseases is unknown.

The patient of Case 1 experienced CRPS symptom improvement within 4 weeks after starting LDN, including a cessation of his dystonic spasms in his upper extremities. Although the patient continues to obtain sustained benefit from LDN, chronic underlying drivers may be maintaining this patient's CRPS symptoms. For instance, autoimmune attack on certain neurotransmitter receptors in the peripheral and central nervous system are implicated in CRPS. A recent study found that $90 \%$ of adult CRPS patients had either autoantibodies against the beta- 2 adrenergic receptor, or the M2 muscarinic acetylcholine receptor (Kohr et al. 2011). LDN would not be expected to block the primary steps of an autoimmune attack on these neurotransmitter receptor targets. Instead, LDN would be expected to attenuate proinflammatory reactions of microglia that would likely be activated in a neuroimmune attack.

The fixed dystonias, which frequently occur in CRPS patients, are sometimes viewed as symptoms of a psychogenic illness - a pathology originating in the mind (such as conversion disorder) (Ochoa and Verdugo 1995; Verdugo and Ochoa 2000; Hawley and Weiner 2011). This presumed etiology for CRPS dystonia can restrict treatment options to psychotherapy. Other treatment options are available. Intrathecal baclofen, for instance, is effective for upper extremity CRPS dystonia, but not for lower extremity CRPS dystonia (van Hilten et al. 2000). Deep brain stimulation (DBS) has recently been used for multi-focal dystonia in CRPS patients (Javed et al. 2011). Positive results from these approaches provide evidence that CRPS dystonias are responsive to physiological interventions.

Fixed dystonia in the lower extremity has been very difficult to treat in CRPS patients (van Hilten et al. 2000; Raja 2009). Physical therapy (PT) is a first-line intervention for the fixed dystonias of CRPS, however, the limited range of motion of these dystonias often limit the effectiveness of PT. Botulium toxin injections have been reported to suppress dystonia in the shoulder and upper extremities of CRPS patients (Kharkar et al. 2011a, b). However, there are no publications that report success of botulinum injection therapy in the lower extremities of CRPS patients.

The dystonic lower leg of the CRPS patient in Case 2 required a multi-modal treatment approach. Without reduction of the subluxed fixed dystonic posture, recalcitrant dermal ulcerations, and/or focal osteopenia might have resulted from interrupted blood flow to the affected extremity. Sublingual ketamine and low-dose naltrexone were given prior to casting, 
to reduce the risk of CRPS symptom spread, and to reduce central sensitization, which was presumed to be already present in the patient's nervous system.

Moderate reduction of pain in fibromyalgia patients takes place within days after treatment with LDN (Younger and Mackey 2009). For the two patients described in this current report, improvement of CRPS symptoms occurred within 2-4 weeks after receiving LDN. In CRPS patients, pain may require longer periods of time to subside, because CRPS patients have multiple interlocking and overlapping pathophysiologies, including structural damage to peripheral tissues (e.g. focal loss of bone density, small fiber neuropathies, and vascular damage) (Fig. 1) (Moriwaki et al. 1997; Oaklander and Fields 2009).

Remission of fixed dystonia, along with other CRPS symptoms, such and allodynia and vasomotor abnormalities, could be readily interpreted as a physiological response linked to LDN therapy, as opposed to effects produced by psychological suggestion. However, it is clear that a randomized, blinded clinical trial is needed to exclude a psychogenic mechanism of action of LDN in CRPS patients. CRPS patients do have unusual psycho-active nociceptive, autonomic, and motor symptoms, which can be provoked by psycho-perceptional conflicts (Cohen et al. 2012; Bailey et al. 2012). LDN may be a useful tool to determine whether these psycho-active symptoms of CRPS patients are indicative of functional neurological disorders, which may be secondary to neuroinflammatory lesions in the CNS (Cooper and Clark 2012).

\section{Conclusion}

Our use of LDN treatment for CRPS patients was motivated by a presumed neuroinflammatory etiology for longstanding CRPS symptoms. The remission of pain and dystonic spasms in Case 1, as well a remission of all CRPS symptoms (including fixed dystonia) in Case 2, provide evidence that a multi-modal interventional approach, which includes low-dose naltrexone (a known glial attenuator), should be considered as a treatment option for the treatment of CRPS patients, particularly those patients with dystonic movement disorders.

\section{Acknowledgments We are grateful to our patients for their cooperation} and assistance.

Conflict of interest The authors state that they do not have any conflict of interest.

Open Access This article is distributed under the terms of the Creative Commons Attribution License which permits any use, distribution, and reproduction in any medium, provided the original author(s) and the source are credited.

\section{References}

Bailey J, Nelson S, Lewis J, McCabe CS (2012) Imaging and clinical evidence of sensorimotor problems in CRPS: utilizing novel treatment approaches. J Neuroimmune Pharmacol (Epub ahead of print)

Barbosa IG, Rocha NP, Huguet RB, Ferreira RA, Salgado JV, Carvalho LA, Pariante CM, Teixeira AL (2012) Executive dysfunction in euthymic bipolar disorder patients and its association. J Affect Disord 137:151-155

Besedovsky HO, del Rey A (2011) Central and peripheral cytokines mediate immune-brain connectivity. Neurochem Res 36:1-6

Binkley K (2012) Improving the diagnosis and treatment of CRPS: insights from a clinical immunologist's personal experience with an underrecognized neuroinflammatory disorder. J Neuroimmune Pharmacology (Epub ahead of print)

Birklein F, Riedl B, Sieweke N, Weber M, Neundörfer B (2000) Neurological findings in complex regional pain syndromesanalysis of 145 cases. Acta Neurol Scand 101:262-269

Church AJ, Dale RC, Giovannoni G (2004) Anti-basal ganglia antibodies: a possible diagnostic utility in idopathic movement disorders? Arch Dis Child 89:611-614

Cohen HE, Hall J, Harris N, McCabe CS, Blake DR, Jänig W (2012) Enhanced pain and autonomic responses to ambiguous visual stimuli in chronic Complex Regional Pain Syndrome (CRPS) type I. Eur J Pain 16:182-195

Cooper MS (2011) Nerve injuries and the fixed dystonias of CRPS. Pain Med 12:842-843

Cooper MS, Clark VP (2012) Neuroinflammation, neuroautoimmunity, and the co-morbidities of CRPS. J Neuroimmune Pharm (Epub ahead of print)

Coull JA, Beggs S, Boudreau D, Boivin D, Tsuda M, Inoue K, Gravel C, Salter MW, de Koninck Y (2005) BDNF from microglia causes the shift in neuronal anion gradient underlying neuropathic pain. Nature 15:1017-1021

Del Valle L, Schwartzman RJ, Alexander G (2009) Spinal cord histopathological alterations in a patient with longstanding complex regional pain syndrome. Brain Behav Immun 23:85-91

Fellner L, Irschick R, Schanda K, Reindl M, Klimaschewski L, Poewe W, Wenning GK, Stefanova N (2013) Toll-like receptor 4 is required for $\alpha$-synuclein dependent activation of microglia and astroglia. Glia 61:349-60

Fong CY, de Sousa C (2006) Childhood chorea-encephalopathy associated with human parvovirus B19 infection. Dev Med Child Neurol 48:526-528

Grillo E, da Silva RJ (2009) Childhood chorea-encephalopathy and unremarkable MRI: an association suggesting parvovirus B19 infection. Dev Med Child Neurol 51:759-761

Hawley JS, Weiner WJ (2011) Psychogenic dystonia and peripheral trauma. Neurology 77:496-502

Hutchinson MR, Zhang Y, Brown K, Coats BD, Shridhar M, Sholar PW, Patel SJ, Crysdale NY, Harrison JA, Maier SF, Rice KC, Watkins LR (2008) Non-steroselective reversal of neuropathic pain by naloxone and naltrexone: involvement of toll-like receptor 4 (TLR4). Eur J Neurosci 28:20-29

Javed S, Sharples PM, Khan S, White E, Gill S (2011) Recovery from fixed dystonia in complex regional pain syndrome type 1 (CRPS1) following deep brain stimulation (DBS) surgery. Arch Dis Child 96(Suppl 1):A81

Kaindl AM, Degos V, Peineau S, Gouadon E, Chhor V, Loron G, Le Charpentier T, Josserand J, Ali C, Vivien D, Collingridge GL, Lombet A, Issa L, Rene F, Loeffler JP, Kavelaars A, Verney C, Mantz J, Gressens P (2012) Activation of microglial $\mathrm{N}$-methyl-D-aspartate receptors triggers inflammation and neuronal cell death in the developing and mature brain. Ann Neurol 72:536-49 
Kharkar S, Ambady P, Venkatesh Y, Schwartzman RJ (2011a) Intramuscular botulinum toxin in complex regional pain syndrome: case series and literature review. Pain Phys 14:419-24

Kharkar S, Ambady P, Yedatore V, Schwartzman RJ (2011b) Intramuscular botulinum toxin A (BtxA) in complex regional pain syndrome. Pain Phys 14:311-316

Kohr D, Singh P, Tschernatsch M, Kaps M, Pouokam E, Diener M, Kummer W, Birklein F, Vincent A, Goebel A, Wallukat G, Blaes F (2011) Autoimmunity against the $b(2)$ adrenergic receptor and muscarinic-2 receptor in complex regional pain syndrome. Pain 152:2690-2700

Kolb L, Lang C, Seifert F, Maihöfner C (2012) Cognitive correlates of "neglect-like syndrome" in patients with complex regional pain syndrome. Pain 153:1063-1073

Leow-Dyke S, Allen C, Denes A, Nilsson O, Maysami S, Bowie AG, Rothwell NJ, Pinteaux E (2012) Neuronal toll-like receptor 4 signaling induces brain endothelial activation and neutrophil transmigration in vitro. J Neuroinflammation 9:230

Linnman C, Becerra L, Borsook D (2012) Inflaming the brain: CRPS a model disease to understand neuroimmune interactions in chronic pain. J Neuroimmune Pharmacology (Epub ahead of print)

Liu B, Du L, Hong JS (2000) Naloxone protects rat dopaminergic neurons against inflammatory damage through inhibition of microglia activation and superoxide generation. J Pharmacol Exp Ther 293:607-617

Maihöfner C, Peltz E (2011) CRPS, the parietal cortex and neurocognitive dysfunction: an emerging triad. Pain 152:1453-1454

Maihöfner C, Handwerker HO, Neundörfer B, Birklein F (2003) Patterns of cortical reorganization in complex regional pain syndrome. Neurology 61:1707-1715

Maihöfner C, Handwerker HO, Neundörfer B, Birklein F (2004) Cortical reorganization during recovery from complex regional pain syndrome. Neurology 63:693-701

Mao-Ying QL, Wang XW, Yang CJ, Li X, Mi WL, Wu GC, Wang YQ (2012) Robust spinal neuroinflammation mediates mechanical allodynia in Walker 256 induced bone cancer rats. Mol Brain 5:16

Marinus J, Moseley GL, Birklein F, Baron R, Maihöfner C, Kingery WS, van Hilten JJ (2011) Clinical features and pathophysiology of complex regional pain syndrome. Lancet Neurol 10:637-648

Milligan ED, Watkins LR (2009) Pathological and protective roles of glia in chronic pain. Nat Rev Neurosci 10:23-36

Mitchell SW (1872) Injuries of nerves and their consequences. J.B. Lippincott \& Company

Mitchell MC, Memisoglu A, Silverman BL (2012) Hepatic safety of injectable extended-release naltrexone in patients with chronic hepatitis C and HIV infection. J Stud Alcohol Drugs 73:991-997

Moriwaki K, Yuge O, Tanaka H, Sasaki H, Izumi H, Kaneko K (1997) Neuropathic pain and prolonged regional inflammation as two distinct symptomatological components in complex regional pain syndrome with patchy osteoporosis - a pilot study. Pain 72:277-282

Oaklander AL, Fields HL (2009) Is reflex sympathetic dystrophy/ complex regional pain syndrome type I a small-fiber neuropathy? Ann Neurol 65:629-638

Ochoa JL, Verdugo RJ (1995) Reflex sympathetic dystrophy. A common clinical avenue for somatoform expression. Neurol Clin $13: 351-363$
Raja SN (2009) Motor dysfunction in CRPS and its treatment. Pain $143: 3-4$

Rea F, Bell JR, Young MR, Mattick RP (2004) A randomized, controlled trial of low dose naltrexone for the treatment of opioid dependence. Drug Alcohol Depend 75:79-88

Rolan P, Hutchinson M, Johnson K (2009) Ibudilast: a review of its pharmacology, efficacy and safety in respiratory and neurological disease. Expert Opin Pharmacother 10:2897-2904

Sansing LH, Tüzün E, Ko MW, Baccon J, Lynch DR, Dalmau J (2007) A patient with encephalitis associated with NMDA receptor antibodies. Nat Clin Pract Neurol 3:291-296

Schouten AC, van de Beek WJ, van Hilten JJ, van der Helm FC (2003) Proprioceptive reflexes in patiens with reflex sympathetic dystrophy. Exp Brain Res 151:1-8

Schwartzman RJ, Erwin KL, Alexander GM (2009) The natural history of complex regional pain syndrome. Clin J Pain 25:273-280

Schwenkreis P, Janssen F, Rommel O, Pleger B, Völker B, Hosbach I, Dertwinkel R, Maier C, Tegenthoff M (2003) Bilateral motor cortex disinhibition in complex regional pain syndrome (CRPS) type I of the hand. Neurology 61:515-519

Sibanc B, Lesnicar G (2002) Complex regional pain syndrome and lyme borreliosis: two different diseases? Infection 30:396-399

Sudeck PHM (1901) Uber die akute (reflektorische) Knochenatrophie nach Entzundungen und Verletzungen an den Extremitaten und ihre klinischen Erscheinungen. Fortschr Geb Rontgenstr 5:277

Tanga FY, Nutile-McMenemy N, DeLeo JA (2005) The CNS role of Tolllike receptor 4 in innate neuroimmunity and painful neuropathy. Proc Natl Acad Sci U S A 102:5856-5861

van de Warrenburg BPC, Cordivari C, Brown P, Bhatia KP (2007) Persisting hyperekplexia after idiopathic, self-limiting brainstem encephalopathy. Mov Disord 22:1017-1020

van Hilten JJ (2010) Movement disorders in complex regional pain syndrome. Pain Med 11:1274-1277

van Hilten BJ, van de Beek WJ, Hoff JI, Voormolen JH, Delhass EM (2000) Intrathecal baclofen for the treatment of dystonia in patients with reflex sympathetic dystrophy. $\mathrm{N}$ Engl J Med 343:625-630

van Hilten JJ, van de Beek WJ, Vein AA, van Dijk JG, Middlekoop HA (2001) Clinical aspects of multifocal or generalized tonic dystonia in reflex sympathetic dystrophy. Neurology 56:1762176

van Hilten JJ, Rooijen DE, Geraedts EJ, Marinus J, Jankovic J (2011) Peripheral trauma and movement disorders: a systematic review of reported cases. J Neurol Neurosurg Psychiatry 82:892-898

van Rijn MA, Marinus J, Putter H, Bosselar SR, Moseley GL, van Hilten JJ (2011) Spreading of complex regional pain syndrome: not a random process. J Neural Transm 118:1301-1309

Verdugo RJ, Ochoa JL (2000) Abnormal movements in complex regional pain syndrome. Muscle Nerve 23:198-205

von Hehn C, Baron R, Woolf CJ (2012) Deconstructing the neuropathic pain phenotype to reveal neural mechanisms. Cell 73:638-651

Watkins LR, Hutchinson MR, Rice KC, Maier SF (2009) The "toll" of opioid-induced glial activation: improving the clinical efficacy of opioids by targeting glia. Trends Pharmacol Sci 30:581-591

Younger J, Mackey S (2009) Fibromyalgia symptoms are reduced by low-dose naltrexone: a pilot study. Pain Med 10:663-672 